\title{
EVALUATION OF BONE HEALING IN FEMURS LENGTHENED VIA THE GRADUAL DISTRACTION METHOD
}

\author{
Jiri Jochymek ${ }^{\text {a*}}$, Petr Gal ${ }^{\mathrm{a}}$ \\ a Department of Pediatric Surgery, Orthopedics, and Traumatology (DPSOT) of the Children's Medical Center (CMC), \\ Faculty Hospital in Brno, and Medical Faculty of the Masaryk University in Brno \\ e-mail:jjochymek@seznam.cz
}

Received: April 2, 2007; Accepted (with revision): May 24, 2007

Key words: Leg lengthening/Gradual distraction/External fixation/Leg discrepancy/Complication

Background: Treatment of leg length inequality via lengthening of the shorter extremity is an infrequent orthopedic procedure due to the requirement of special distraction devices and possible serious complications. Essential qualitative changes in operative technique development are associated with the name of G. A. Ilizarov, who paved the way for the autoregenerate gradual distraction method in the 1950s.

Material and Methods: In the years 1990 through 2006 a total of 57 patients underwent femur lengthening via gradual distraction using various types of external fixators at the Department of Pediatric Surgery, Orthopedics, and Traumatology, Faculty Hospital in Brno. The quality of bone healing was monitored and a number of parameters followed and statistically evaluated using regularly scheduled X-ray examinations.

Results: In 11 cases we had to remove the external fixator following the distraction phase, perform an osteosynthesis via a splint and fill the distraction gap via spongioplasty. The bone healing was satisfactory in the remaining 46 patients and the lengthened bone required no other fixation method. The analysis showed statistically significant deceleration in bone healing following distraction in female patients over 12 years of age, and in boys over 14 years of age. Lack of periosteal callus five weeks after surgery always signified serious problems in further healing. Severe complications were recorded in 11 cases during the distraction phase, and in 9 cases after the removal of the distraction apparatus.

Conclusions: The aim of this report was to present the results of our study of distraction gap bone healing using the gradual lengthening approach.

\section{INTRODUCTION}

Equalization of uneven leg length via prolongation of the shorter extremity is usually preferred by both the patients and their parents. However, this method can not be used in all cases. Femoral lengthening via gradual distraction is one of the less frequent procedures in orthopedics due to its demand factor and high percentage of complications. Therefore, these procedures should be reserved for specialized orthopedics centers involved in such complex problems on a regular basis. The Department of Pediatric Surgery, Orthopedics, and Traumatology (DPSOT, formerly the Pediatric Orthopedics Clinic) of the Children's Medical Center (CMC), Faculty Hospital in Brno (referred to as "our department" in further text) has been involved in leg lengthening procedures since 1965 and at present is routinely performing length corrections of both upper and lower extremities ${ }^{1}$.

An essential qualitative shift in approach to this issue is connected with the name of Ilizarov, who introduced the original method of subperiosteal corticotomy in 1952 in the location of the diaphysis or the metadiaphyseal region followed by gradual distraction of the autoregenerate. Corticotomy with maximal preservation of the peri- osteum, the endosteum, and the bone marrow, nowadays more frequently performed via the open approach, supports the formation of the regenerated bone, which is apparent throughout the whole distraction phase. Sufficient amount of bone tissue is usually produced during distraction. The distraction apparatus is removed after clinically and X-ray ascertained strengthening of the osteal regenerate, which on an average approximately doubles the time of the lengthening itself ${ }^{2,3}$.

When performing extremity lengthening we follow these rules and principles. All patients in this study were treated via the gradual distraction lengthening method.

The aim of this paper is to present our results with femoral lengthening in a large group of patients, perform a basic analysis of bone healing in the distraction gap and describe several major factors influencing the quality of the originating autoregenerate.

\section{MATERIAL AND METHODS}

The study included a group of 57 patients treated with femoral lengthening at our department during the years 1990 through 2006. The group consisted of 31 male 
and 26 female patients in ages ranging from 6 years and 2 months up to 16 years and 8 months (average 12 years and 4 months). In 18 patients (31.5\%) the shortening of the leg was secondary, most frequently due to posttraumatic injuries or developmental dysplasia of the hip (DDH). The remaining 39 patients (68.5\%) suffered from primary idiopathic shortening of the lower extremity.

The leg shortening average in our group was $55 \mathrm{~mm}$ with a maximal shortening of $90 \mathrm{~mm}(30 \mathrm{~mm}-90 \mathrm{~mm})$.

The distraction devices applied were the external Ilizarov fixator in 2 cases, a device allowing for simultaneous distraction on two levels in one case, an Orthofix in 7 cases, external Mefisto fixator in 17 cases, and the Wagner apparatus in the remaining 30 cases, respectively.

Following the standard installation of the external fixator to the thigh a transverse or oblique diaphyseal osteotomy of the femur was performed using the Gigli saw in 5 cases, and an oscillating saw in all remaining cases.

The interval from surgery to lengthening initiation reached an average of 8 days (3-12 days). The average speed of lengthening was approximately $1 \mathrm{~mm} /$ day with 1 turn per day in the majority of patients, and half of a turn twice a day in 7 patients. The usual lengthening schedule consisted of 5 days of distraction followed by 2 days of stillstand.

The quality of bone healing was monitored via X-ray pictures, in which the periosteal callus, endosteal callus, and filling of the distraction gap with homogeneous bone substance were evaluated. Spongialization, bone remodeling, corticalis appearance, and bone marrow canal emergence were also monitored on the radiographs. All of these data were statistically evaluated. Other observed parameters included the size of the original shortening, sex of the patient, and age at the time of surgery. For assessment of the influence of patient age on the bone healing the patients were divided into 1-year age groups.

Some of the parameters were statistically evaluated in connection with the healing index ${ }^{4-8}$, a generally accepted bone healing quantifying assessment. The healing index represents the time in which the newly formed bone of the regenerate becomes robust and consolidated enough to allow for removal of the external fixator. For many years the quality of bone healing has been also expressed at our department via a so-called load index, which states the time from surgery to the point where the patient walks without the use of crutches, recalculated to $1 \mathrm{~cm}$ of lengthening obtained $^{1}$.

Statistical evaluation of most factors was performed via a parametric or in case of groups with non-Gaussian data distribution a non-parametric test (parametric Student's T-test, non-parametric Mann-Whitney U-test and chi-square test, etc.). Part of the data were analyzed using the Microsoft Excel software, while crucial statistical analyses were performed employing the NCSS 2000 system (L. Hintze, Kaysville, Utah, USA).

\section{RESULTS}

The ultimate femur lengthening in our group of patients reached an average of $45 \mathrm{~mm}$, with a minimum of $20 \mathrm{~mm}$ and maximum of $85 \mathrm{~mm}$, respectively. The relative lengthening of the bone ranged from $5.1 \%$ to $40.2 \%$.

In 11 cases we were forced to remove the external fixation device following the distraction phase, perform an osteosynthesis via a splint, and fill up the distraction gap with an autologous or allogeneic spongioplasty. This was necessary especially in relatively older patients, as all of these 11 children were 12 years of age or older. The main reason for this approach, considered non-typical by Ilizarov, was insufficient bone callus generation.

The bone healing was satisfactory in the remaining 46 patients and the lengthened bone required no other method of fixation.

The quality of bone healing was monitored via regularly scheduled radiographs. The periostal callus represented a first sign of bone healing in patients with subperiosteal femoral osteotomy. It appeared in an average of 2.5 weeks (1.5-7 weeks) following the surgery and its absence in week 5 after surgery signified poor bone healing. In all patients requiring consequent osteosynthesis the first signs of periosteal callus appeared 5 weeks or later after surgery.

The endosteal callus production was recorded in an average of 6 weeks (4-13 weeks) following surgery. Homogeneous filling of the bone gap was observed in the fourth month after surgery (3-6 months).

Spongialization of the osteal fragments was recorded in an average 5.5 months (4.2-8.1 months), and the bone marrow canal formation was observed in 15.2 months (12.8-17.5 months) following surgery, respectively.

Normal shape and structure of the lengthened bone were observed in an average of 23.1 months (21.726.2 months) from surgery. In the 11 cases treated with added splinter and spongioplasty the production of normal bone structure required an additional 7.2 months (5.9-9.1 months).

Full load to the treated extremity was allowed an average of 10.1 months (8.9-12.4 months) after distraction termination based on adequate X-ray results. (Tab. 1)

No statistically significant influence of the sex of the patient on bone healing was observed, as this factor did not affect the healing index. A direct dependence between age at the time of surgery and bone healing was detected, although the difference becomes statistically significant only after 12 years of age in girls (versus younger female patients) and after 14 years of age in boys (versus younger male patients), as the bone healing process decelerates in the older patient groups. The healing index is significantly worse in girls over 12, and boys over 14 years of age, respectively.

The relationship between patient age and load index was analyzed after dividing the patients into subgroups by one year of age. Statistically significant differences were recorded between all age groups always in favor of the younger patient group. In other words, the load index data 
Table 1. Average and standard deviation, minimal, maximal values of monitored bone healing parameters $(n=57)$.

\begin{tabular}{|l|c|c|}
\hline Periosteal callus (weeks) & $2.5 \pm 0.7$ & $(1.5-7)$ \\
\hline Endosteal callus (weeks) & $5.8 \pm 1.5$ & $(4-13)$ \\
\hline Homogeneous gap filling (months) & $3.9 \pm 0.8$ & $(3-6)$ \\
\hline Spongialization (months) & $5.5 \pm 1.1$ & $(4.2-8.1)$ \\
\hline Bone marrow canal (months) & $15.2 \pm 2.1$ & $(12.8-17.5)$ \\
\hline Normal bone shape (months) without spongioplasty ( $\mathrm{n}=46)$ & $23.1 \pm 1.2$ & $(21.7-26.2)$ \\
\hline Normal bone shape (months) with spongioplasty $(\mathrm{n}=11)$ & $30.3 \pm 1.4$ & $(28.1-33,9)$ \\
\hline Full load (months from termination of distraction) & $10.1 \pm 0.9$ & $(8.9-12.4)$ \\
\hline
\end{tabular}

Table 2. The influence of age on the healing index and the load index.

\begin{tabular}{|c|c|c|c|c|c|}
\hline $\begin{array}{c}\text { Girls (n= 26) } \\
\text { Age (years) }\end{array}$ & Healing Index & $\begin{array}{c}\text { Load } \\
\text { Index }\end{array}$ & $\begin{array}{c}\text { Boys (n=31) } \\
\text { Age (years) }\end{array}$ & Healing Index & $\begin{array}{c}\text { Load } \\
\text { Index }\end{array}$ \\
\hline $6-7$ & - & + & $6-7$ & - & + \\
\hline $8-9$ & - & + & $8-9$ & - & + \\
\hline $10-11$ & - & + & $10-11$ & - & + \\
\hline $12-13$ & + & + & $12-13$ & - & + \\
\hline $14-15$ & + & + & $14-15$ & + & + \\
\hline $16-17$ & + & + & $16-17$ & + & + \\
\hline
\end{tabular}

- No statistically significant difference in comparison with the previous age group was found

+ Statistically significant difference in comparison with the previous age group was found

was significantly better in each younger age group when compared to older age groups. (Tab. 2)

No statistically significant differences in bone healing were recorded comparing various types of external fixation devices.

Other factors possibly influencing the quality of bone healing (time interval from surgery to distraction initiation, pace of distraction, resulting distraction, etc.) could not be statistically analyzed since our group of patients was statistically homogeneous in these parameters.

In 20 patients out of the total of $57(35.08 \%)$ complications were recorded, out of which 11 occurred during the distraction phase and the remaining 9 after removal of the distraction apparatus.

During the distraction phase we have detected 4 cases of fragment angulation (7.01\%), 2 cases of deep inflammation $(3.50 \%), 2$ cases of joint subluxation $(3.50 \%)$, 2 cases of severe restriction of knee joint movement $(3.50 \%)$, and 1 neurological disorder with a subsequent nerve revision ( $1.75 \%)$.

Following the removal of the external fixator we have recorded 5 fractures $(8.77 \%)$ and 2 stress fractures in the prolongation region $(3.50 \%)$, and 2 cases of angulation of the lengthened femur $(3.50 \%)$.

\section{DISCUSSION}

The surgical approach to significant lower extremity length inequality can be principle divided to procedures altering the longer extremity, i.e. bone shortening surgery, and to procedures altering the shorter extremity, i.e. bone lengthening surgery.

Currently, a uniphase shortening in the proximal femur region is used in the bone shortening operations. Surgery on the crus was abandoned due to a number of serious healing complications and for esthetic reasons. Procedures based on stopping of the bone growth fall into this category as well. In the 12 trough 14 years age group we can utilize the growth potency of the shorter extremity by intercepting the growth of the longer and faster growing extremity via so-called Blount staples (clips).

Uniphase extremity prolongation have been all but abandoned, perhaps with the exception of surgery on the pelvis.

Gradual prolongation methods represent the surgical approach of choice in this area, either via the controlled distraction epiphyseolysis or via gradual distraction of the newly formed regenerate, which was the method used in this study. 
The gradual distraction approach is the most elegant method of unequal leg length correction, preferred in most cases by both the patients and their parents. Even though the method has been known since the 1930s, a fundamental qualitative change in this method will be forever connected with the Russian orthopedic surgeon Ilizarov. His work was later expanded by DeBastiani, Monticelli and Spinelli, and other authors, who further developed the leg lengthening method over the last twenty years ${ }^{4,5,6,8}$.

In spite of marked progress in the area this therapeutic approach is not frequently used and remains burdened with a high complication rate. The complication incidence data in literature varies significantly. With the exception of the rare $0-40 \%$ rate published by DeBastiani ${ }^{4,7,8}$, the complication incidence in our group of patients was fully comparable with data from other authors ${ }^{1,6,9,10}$. While some authors divide the complications of the gradual distraction approach to minor and major ${ }^{7,10}$, we preferred a division into complications occurring during the distraction phase versus complications occurring after the removal of the distraction device ${ }^{1,11}$. As for the monitoring of the actual bone healing, the results in our group of patients are in accordance with previously published data. We agree with the notion that insufficient attention is usually paid to periosteal callus monitoring ${ }^{11,12}$. Our experience shows that the first occurrence of the periosteal callus later than 3 weeks after surgery represents an initial indication of poor healing, while the first occurrence of the periosteal callus later than 5 weeks after surgery signalizes a probable need for osteosynthesis and spongioplasty. In all 11 patients of our group requiring osteosynthesis and spongioplasty the periosteal callus failed to form prior to week 5 following the original surgical procedure.

Monitoring of both the periosteal callus and the endosteal callus informs us about the bone healing capacity of the individual patient, allowing us to adjust the lengthening pace, verify the quality of the external fixation, and prepare for the possible necessity of future operations. The extent of the resulting bone prolongation certainly affects the bone healing process ${ }^{1,7,11}$. However, we were not able to sufficiently attest this premise ourselves.

Nevertheless, our results fully correspond with the data and experience of other authors who do not allow their patients to fully stress the lengthened lower extremity earlier than 1 year after prolongation completion ${ }^{8,11,12,13}$.

The question of optimal patient age for femur lengthening receives significant attention in the literature. The majority of authors demonstrate a direct relation between bone healing and the age of the patient. Some authors state a demonstrably poor healing within the distraction gap after 10 years of age. However, these assertions are mostly based on experience rather than exact statistical analyses ${ }^{1,11,12}$.

Our results reflect this generally accepted claim. Specifically, the bone healing was significantly decelerated after 12 years of age in female patients, and after 14 years of age in male patients, respectively. We have reached these results evaluating the internationally recognized healing index ${ }^{4-8}$. However, we realize that the healing index works closely with the time of distraction device removal, which depends on subjective evaluation of the callus by the attending physician. Our teachers, who followed the leg lengthening issues closely at our clinic in the past, therefore recommended the calculation of a socalled load index ${ }^{1}$, which more truly predicates the quality of the healing within the prolonged bone, since this index considers the complete time period critical for the patient ${ }^{1}$. The statistical analysis of patient age in regard to the load index shows a significant difference between all age groups in our study, thus demonstrating the beneficial effect of lower age on the bone healing process.

Analysis of the influence of sex of the patient on bone healing led us to a conclusion corresponding with previously published data. If we disregard the age factor, the sex of the patient itself has no significant effect on the bone healing process.

Other factors certain to affect bone healing in the distraction gap such as the time interval from surgery to distraction initiation or pace of distraction could not be statistically analyzed in our group of patients. The pace of distraction used in our group of patients (approximately $1 \mathrm{~mm}$ per day) followed the generally accepted norm, as did the time interval from surgery to distraction initiation. We agree with the notion of Ilizarov that the interval between surgery and start of the femur distraction should not be shorter than 5 to 7 days ${ }^{1-3,6,7,8,13,14}$.

\section{CONCLUSION}

The aim of our publication was to present to the professional public the results of our study of distraction gap bone healing using the gradual lengthening approach. In a relatively large group of 57 patients we monitored the individual bone healing stages during femoral lengthening via radiographs plus clinical examinations and we observed factors influencing the bone healing process.

The results of our study show statistically significant deceleration in bone healing in female patients over 12 years of age versus younger girls, and a similar healing deceleration in male patients of 14 years of age and older versus younger boys. Another clinically relevant conclusion is the fact that a lack of periostal callus five weeks after surgery signifies serious bone healing impairment, requiring osteosynthesis and spongioplasty in further care.

Our findings may help in planning the therapeutic approach in patients with unequal leg length, especially if the treatment of choice is the gradual femoral distraction method.

\section{REFERENCES}

1. Janovec M. Prodlužování dolních končetin. Praha: Avicenum 1984

2. Ilizarov GA, Deviatov M. Operative elongation of the leg. Ortop Travmatol Protez 1971; 32:20-25.

3. Ilizarov GA. The principles of the Ilizarov method. Bull Hosp Jt Dis Orthop Inst 1988; 48:1. 
4. DeBastiani G, Aldegheri R, Renzi-Brev L, et al. Limb lengthenings by callus distraction. J Pediatr Orthop 1987; 7:129-134.

5. Monticelli G, Spinelli R. Leg lengthening by closed metaphyseal corticotomy. Ital J Orthop Traumatol 1983; 4:139.

6. Dungl P, et al. Ortopedie. Grada Publishing a.s. 2005.

7. Tachdjian MO. Pediatric Orthopedics. Vol. I-IV. $2^{\text {nd }}$ ed. Philadelphia: W. B. Saunders, 1990.

8. Herzenberg JE, Waanders NA. Calculating rate and duration of distraction with the Ilizarov technique. Orthop Clin North Am 2002; 33:601.

9. Canadel J, dePablos J. Methods of bone lengthening and their applications. Editicia de Bibliotecas. Universidad de Navara: Pamplona 1989
10. Eldridge JC, Bell DF. Problems with substantial limb lengthening. Orthop Clin North Am 2001; 32:625-631.

11. Janovec M, Polách J. Rozbor výsledku prodlužování femuru u 34 dětí a dospívajících. Acta chir orthop traum Čech, 57, 1990; 5:405416.

12. Paley D. Current techniques of limb lengthening. J pediatr orthop, 8, 1988; 1:73-92

13. Pouliquen JC et al. Allongement de fému chez l' enfant et l'adolescent. Rev Chir Orthop 1999; 85:239-251.

14. Monticelli G, Spinelli R. Distraction epiphyseolysis as a method of limb lengthening. III. Clinical Application. Clin Orthop 1991; 154:274-285. 